\title{
Study on the k-Hilfer-Prabhakar fractional viscoelastic model considering time varying damage
}

\author{
Yiying Feng ${ }^{1}$, Xiao-Jun Yang ${ }^{2}$, Jian-Gen Liu ${ }^{1}$, and Zhan-Qing Chen ${ }^{1}$ \\ ${ }^{1}$ China University of Mining and Technology \\ ${ }^{2}$ China University of Mining and Technology State Key Laboratory for Geomechanics and \\ Deep Underground Engineering
}

October 15, 2020

\begin{abstract}
The element model of the viscoelastic constitutive model has its significant advantage in describing the three stages of rheological curve. Through the medium of the k-Hilfer-Prabhakar fractional derivative, our primary intension in this paper is to establish a viscoelastic constitutive model coupling with the nonlinear time varying elastic element $\mathrm{E}(\mathrm{t})$. The k-Hilfer-Prabhakar fractional derivative is characterized by the recovery between the modified model and the known classical models of linear viscoelasticity. According to the theory of the Laplace transform and Boltzmann superposition principle, the strain of the proposed model is obtained. In summing up it may be stated that the analysis of parameter identification indicates the validity and rationality of the modified model.
\end{abstract}

\section{Hosted file}

Study on the k-H-P fractional viscoelastic model considering damage.pdf available https://authorea.com/users/367533/articles/486913-study-on-the-k-hilfer-prabhakarfractional-viscoelastic-model-considering-time-varying-damage

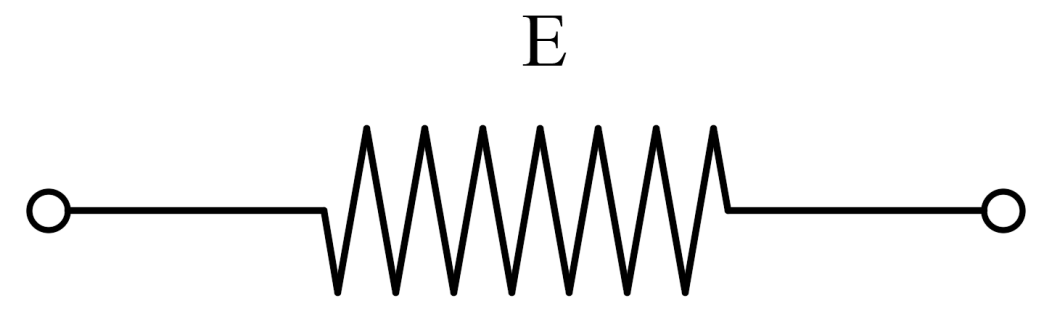


$\eta$
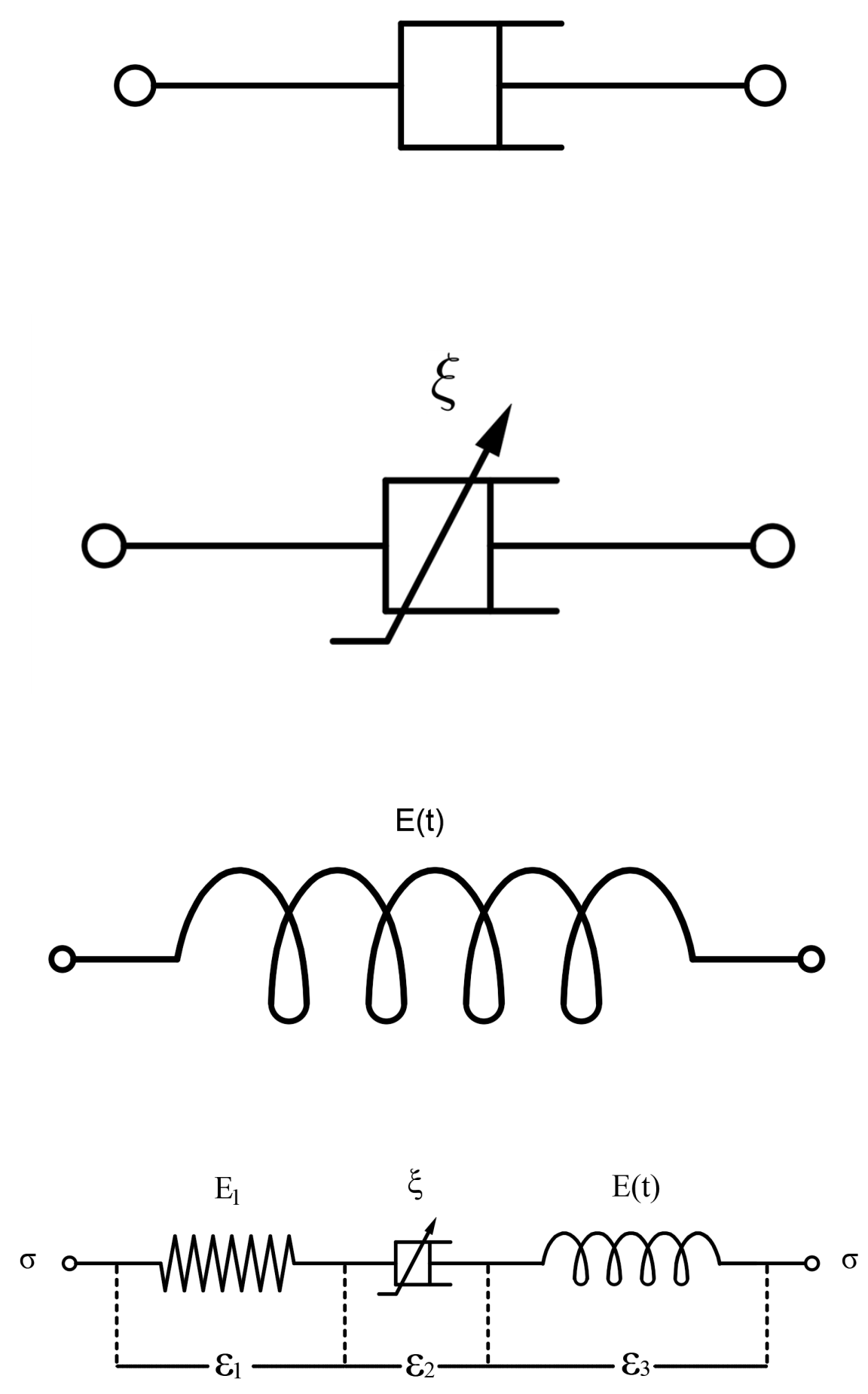
figures/data/data-eps-converted-to.pdf 
figures/nihe/nihe-eps-converted-to.pdf 